\title{
Practice nurse health checks for adults with intellectual disabilities: a cluster-design, randomised controlled trial
}

\author{
Sally-Ann Cooper, Jill Morrison, Linda M Allan, Alex McConnachie, Nicola Greenlaw, \\ Craig A Melville, Marion C Baltzer, Laura A McArthur*,Claire Lammie, Gordon Martin, \\ Eleanor A D Grieve, Elisabeth Fenwick
}

*Ms McArthur died in April, 2013

Institute of Health and Wellbeing, Mental Health and Wellbeing group (Prof S-A Cooper MD, L M Allan RNLD, C A Melville MD, M C Baltzer MA, L A McArthur BSc, C Lammie MA), General Practice and Primary Care group (J Morrison PhD), Robertson Centre for Biostatistics (A McConnachie PhD, N Greenlaw PhD), and Health Economics and Health Technology Assessment group (E A D Grieve MPH, Prof E Fenwick PhD), University of Glasgow, Glasgow, UK; NHS Greater Glasgow and Clyde, Glasgow, UK (G Martin, MB ChB)

Correspondence to: Professor Sally-Ann Cooper, Institute of Health and Wellbeing, University of Glasgow, Glasgow G12 0XH, UK. Sally-Ann.Cooper@glasgow.ac.uk

\section{Summary}

Background Adults with intellectual disabilities have substantial health inequalities and poor access to health care. We assessed whether practice nurse-delivered health checks could improve the health of adults with intellectual disabilities compared with standard care.

Methods In this cluster-design, single-blind, randomised controlled trial, we included general practices in Scotland, UK. From June to December, 2011, we randomly assigned (1:1) these general practices to either health checks plus standard care (health-checks group), or standard care only (control group), and we recruited the patients from these practices. Randomisation was done with stratification by number of GPs per practice and number of registered patients with intellectual disabilities $(<20$ or 220). Two research assistants were masked to allocation, and undertook the review of 9 month medical records and interviews. Participants and carers were not masked. The intervention was one health check designed especially for people with intellectual disabilities delivered by a practice nurse. The objective was improvement in health and health care 9 months after randomisation, and the primary outcome was the incidence of newly detected health needs being met by this timepoint. Whether needs were met was established by the investigators being masked to group allocation. The analysis was by intention to treat. This trial is registered with Current Controlled Trials, number ISRCTN43324841.

Findings Between June 26 and Dec 20, 2011, we recruited 38 practices. 85 participants (from 16 practices) were randomly assigned to intervention and 67 (from 17 practices) to standard care; five of the identified practices did not supply any participants. 83 intervention and 66 standard care participants completed the trial. More newly detected health needs were met in the intervention group than in the control standard care group (median 1 [range 0-8], 76.4\% met [SD 36.5] vs 2 [0-11], $72.6 \%$ met [35.4]; odds ratio [OR] 1.73 [95\% CI 0.93-3.22], $\mathrm{p}=0.085$ ), although this difference was not significant. Significantly more health monitoring needs were met in the intervention group than standard care (median 2 [0-20], 69.9\% [SD 34.2] vs 2 [0-22], 56.8\% [29.4], OR 2.38 [95\% CI 1.314.32, $p=0.0053])$. The probability that health checks are cost effective was between 0.6 and 0.8 , irrespective of the cost-effectiveness threshold level. Costs per patient were $-£ 71.48$ for health checks and $-£ 20.56$ for standard care. The difference $(-£ 50.92)$ was not significant [95\% CI -434 to 362]. No adverse events were attributable to the intervention.

Interpretation Health checks given by practice nurses to adults with intellectual disabilities produced health-care improvements that were more conducive to longer-term health than standard care given to this population. The intervention dominated standard care, being both cheaper and more effective. Health-check programmes might therefore be indicated for adults with intellectual disabilities.

Funding Scottish Government Change Fund, NHS Greater Glasgow and Clyde Research and Development. 


\section{Introduction}

People with intellectual disabilities encounter substantial health inequalities. The standardised mortality ratio for people with moderate to profound intellectual disabilities is 324 in the UK. ${ }^{1}$ This population has very poor health and multimorbidity; eg, point prevalence of mental health disorders for this population globally is $41 \%,{ }^{2}$ problem behaviour $23 \%,{ }^{3}$ epilepsy $25 \%,{ }^{4}$ sensory impairments $50 \%,{ }^{5-8}$ and gastro-oesophageal reflux disorder $50 \% .{ }^{9}$ Swallowing problems, which can cause choking or aspiration, ${ }^{4}$ are common in this population, as are injuries, accidents, and falls. ${ }^{10}$ These health needs have biological, social, and environmental determinants, including poorer access to health care. ${ }^{4,11-15} \mathrm{~A}$ lot of health needs of this population are unmet, and present at more advanced disease stages, with worsened treatment outcomes. Additionally, effects of poor health care accumulate across their lifespan, rather than merely being late-life issues that the general population might experience, which could compound difficulties for the intellectually disabled population over time.

The patterns of ill health, common causes of death, and health-related behaviours in adults with intellectual disabilities differ from the general population. ${ }^{4}$ Smoking and excess alcohol consumption are uncommon, but communication difficulties make these people reliant on carers noticing their problems and taking action. ${ }^{4}$ Background, training, motivation, and understanding of carers, and communication within and across care teams influence whether they notice such problems. By definition, people with intellectual disabilities need additional support in their daily or social functioning. In the UK, such support is typically provided by family carers, or by carers employed by supportproviding organisations who provide care to an individual in their own home, ranging from a few hours per week to $24 \mathrm{~h}$ support every day, depending on the individual's needs. Attitudes and skills of health professionals also affect the support given, and diagnostic overshadowing (the attribution of a person's symptoms and signs to their underlying intellectual disabilities, rather than their additional health needs) is well recognised. There is no National Health Service (NHS) equivalent of paediatric services for adults with intellectual disabilities, so responsibility for their care is given to primary care, within which-for many reasons - it is challenging to address these needs.

The challenges encountered have led to calls for health checks for adults with intellectual disabilities. Health checks for the general population without selection for risk factors or disease are ineffective. ${ }^{16} \mathrm{~A}$ systematic review ${ }^{16}$ suggested that such systematic screening might not be needed because this screening takes place in general practice anyway, and uptake tends to be by people from high socioeconomic status groups who are at decreased risk. However, this systematic review ${ }^{16}$ excluded trials with the high-risk geriatric population. These conclusions are also unlikely to apply to adults with intellectual disabilities, because they are also a high-risk group, with a low use of primary care, and low socioeconomic status. Additionally, general health checks designed for the general population such as the midlife-NHS health check offered in England are not focused on the types of illnesses and disabling disorders that are most prevalent for adults with intellectual disabilities. However, healthcheck evidence related to adults with intellectual disabilities is scarce, with little information from randomised trials, or about health-check costs, acceptability, barriers to uptake, implementation of actions resulting from health checks, or their effect on future health and wellbeing. ${ }^{17}$

A dedicated training route exists to become a registered intellectual disabilities nurse in the UK. This route is a 3 year course that does not require a previous nursing qualification, and is different from the role of nurse practitioners or liaison nurses. A non-randomised intervention study ${ }^{18}$ in Scotland investigated 50 adults with intellectual disabilities who were health checked by intellectual disabilities nurses, matched for sex, age, and ability, and 50 who received treatment as usual (standard care). After 1 year, the intervention group had substantially more health, health monitoring, and healthpromotion needs met, and the intervention was inexpensive. ${ }^{18,19}$ However, the health check took $4 \mathrm{~h}$, and outcomes might have been improved with health checks done by GPs or practice nurses, since the study identified predominantly general health needs. An Australian trial randomly assigned 453 adults receiving paid support from one provider, into 34 clusters. ${ }^{20}$ Improvements in health promotions (eg, sensory impairment testing, immunisations, and cervical screening) were noted in the GP-delivered health-check intervention group 1 year later, but no significant difference in newly detected diseases was noted $(p=0.07) .{ }^{20}$ This intervention was also inexpensive. ${ }^{21}$ Results from another Australian cluster trial that randomly assigned 272 participants to combined health advocacy with the GP-delivered health assessment, or standard care, showed no additional benefits for the intervention. ${ }^{22}$ The extent of health actions from these checks was greater than that recorded in GP records. ${ }^{23}$ However, the health-care system in Australia differs from the that in the UK (eg, patients do not register with a GP), and many organisations provide paid support, so results might not be generalisable. We have not 
identified any trials of health checks for adults with intellectual disabilities delivered by practice nurses rather than GPs. Within the UK, the number of practice nurses delivering specific types of management for long-term disorders-eg, checks for people with diabetes, physical health of people with psychosishas increased, which suggests that this type of management might provide a model to address the health inequalities of adults with intellectual disabilities.

We aimed to identify the clinical and cost-effectiveness of health checks designed for adults with intellectual disabilities and delivered by practice nurses, compared with standard care for these patients.

\section{Methods \\ Trial design and participants}

We used a stratified, cluster, randomised, controlled design. The objective was aimed at the individual level, but we used a cluster design to avoid contamination of intervention effects at general practice level. We recruited GP practices, then enrolled up to ten patients per practice. We invited 102 practices and expected to recruit about 60 practices, but achieved the participant numbers needed after recruiting 38 practices between June 21 and Dec 20, 2011.

We obtained baseline data, then randomly assigned practices to either standard care plus the intervention (health checks delivered by practice nurses), or standard care only. Follow-up was 9 months after randomisation. Participants received $£ 50$ for their time. Practices received $£ 100$ per health check. No changes were made to the trial design during implementation. Unlike England, health checks for adults with intellectual disabilities are not part of the Scottish GP contract and so are not routinely offered; hence the advantage of doing the study in Scotland. Standard care provided by general practice for people with intellectual disabilities in Scotland is the same as that provided for the general population.

We included adults aged 18 years or more with intellectual disabilities who were registered at a participating practice. Exclusion criteria were no valid consent, terminal illness, or their GP regarding the invitation as inappropriate.

MREC-Scotland A and NHS Greater Glasgow and Clyde Research and Development approved the study (10/MRE00/79). Participant consent was in agreement with Scottish law (the Adults with Incapacity [Scotland] Act, 2000), where participants with intellectual disabilities provided written consent if they had the decision-making capacity to do so; otherwise, their welfare guardian or nearest relative provided written consent.

\section{Randomisation and masking}

We randomly assigned whole practices to avoid contamination of intervention effects, because healthchecking some patients could influence practice with others. Practices were stratified by their size (number of GPs: $1-2,3-4$, or $\geq 5$ ), and number of registered patients with intellectual disabilities $(<20$ or $\geq 20)$, in case these factors affected GPs' skill level with this population. The investigation coordinator notified practices and the NHS intellectual disabilities primary care liaison team of group allocation so that they could provide training.

Randomisation was undertaken and checked by staff of the Robertson Centre for Biostatistics who had no other association with the investigation or data management. Practices were randomised to intervention or control in batches; after baseline data had been obtained from a group of practices, they were allocated by identifying all possible sets of allocations that would maintain balance with respect to the stratification variables, and randomly selecting one of these sets. The computer programs and random seeds used to make these allocations were retained, and were stored with the randomisation codes in a separate network location from other study data, with restricted access. Initial statistical programming used dummy randomisation codes. Only after data collection was complete were true randomisation codes released to the statistician.

Two research assistants were masked to allocation, and undertook the review of 9 month medical records and interviews with participants or carers. Practices notified participants or carers whether they would be offered a health check (so they were not masked). Nurses were instructed not to note in 
patients' records that a health check had been done. Consensus meetings research teams were masked to proceedings throughout.

\section{Procedures}

Health checks were delivered by practice nurses at recruited practices, after a training session. The training included communication techniques (including the recognition of pain); identification of common health presentations in this population, the role of carers, and common causes and severity of intellectual disabilities; and hints and tips to maximise the best use of appointment time. Written resources were also provided.

The practices sent appointments, and provided health questionnaires for carers to complete in advance of the health check. The nurses used a health-checking instrument, which is designed for the needs of people with intellectual disabilities, to direct the health check and inform clinical decision making with respect to further standard investigations and treatments. The intervention initiated nurse contact to identify unmet needs. First, people with a carer who could think about, discuss, and record information in advance worked through a carer health check (for people who could not meet this criterion, this information was obtained verbally with the nurse at the health-check appointment). The carer's health check was followed by the health check with the nurse. For health-check details see http://www.gla.ac.uk/researchinstitutes/healthwellbeing/research/mentalhealth/research/projects/heal thchecksforadultswithintellectualdisabiltiesthehechidstudy/

Carer work, followed by a meeting with the nurse, reduces the nurse appointment length. Development of the health-check method was led by the Primary and Community Care Directorate of the Scottish Government. It aims to identify (1) health associations specific to syndromal causes of intellectual disabilities if present, and health needs that are more relevant to the intellectual disabilities population; (2) general health needs (specific symptoms, brief examination, etc); (3) health monitoring needs (ie, chronic diseases that need monitoring or actions), as identified for the GP Quality and Outcomes Framework (QOF), which contains a series of items that GPs should complete within specified timeframes for each of their patients with specified long-term disorders (eg, checking management of diabetes). Performance on the QOF (mostly the portion of completed items) defines the level of renumeration that practices receive for this component of their contract with the UK Government; and (4) health promotion needs (eg, access to population-wide screening programmes, weight management, and education about nutrition).

\section{Outcomes}

The primary outcome was the incidence of new health needs detected and met during the 9 months after randomisation.

Secondary outcomes during the 9 months after randomisation were the extent of health monitoring (QOF items) and health promotion needs being met, the change in participant or carer-rated general health (by use of European Quality of Life-5 Dimensions [EQ- 5D] health-utility scores and Short Form [36] Health Survey [SF-36] scores), serious adverse events, and changes in resource use or costs. No changes were made to outcomes during study implementation.

At baseline and 9 months after randomisation, we extracted data from primary care records about health needs, health-monitoring needs, and health-promotion needs addressed in primary care (eg, influenza immunisation), using a previously developed extraction method. We undertook interviews with participants or carers to complete the client-service receipt inventory, ${ }^{24}$ adverse events information, EQ-5D, SF-36, and health-promotion information not available in GP records, eg, dental appointments. At baseline, ability was assessed with the Vineland Scale. ${ }^{25}$ Two clinician investigators (S-AC and JM) had joint consensus conference meetings to establish whether health needs had been met at baseline and 9 months after randomisation; group allocation was concealed from both these investigators.

\section{Statistical analysis}

For $90 \%$ power and $5 \%$ significance, the previous Scottish intervention study ${ }^{18}$ showed that 51 participants would be needed in each group to detect a rise in the proportion of new health needs being met, from a third to two-thirds (nQuery Advisor v 4.0, based on a continuity-corrected $x^{2}$ test); or 64 in each study group allowing for $20 \%$ attrition. The amount of inflation in sample size needed because of the cluster-randomised design depends on the average cluster size (expected to be 2.5 in this investigation) and intraclass correlation coefficient (ICC). We had no information from which to 
estimate the ICC, but by assuming a value of 0.05 , the increase in sample size needed would be less than $10 \%$. Furthermore, the previous study ${ }^{18}$ used a more detailed health-check instrument, and since then accessibility of primary care might have improved. We therefore cautiously aimed to recruit 75 participants per group.

To determine whether participants were representative of the wider population with intellectual disabilities living in the same geographical area, we compared their characteristics for age, sex, and type of accommodation, by use of information from the primary-care learning-disabilities register for the health-board area.

We adjusted all analyses for sex, age band (18-44,45-64, or >65 years), level of intellectual ability, type of accommodation, and stratification factors. Analyses established any outcome measure differences at 9 months. We included participants in analyses on an intention-to-treat basis. Analyses used generalised linear mixed models, which took into account the cluster design, to estimate the between-group differences, adjusting for the noted covariates. Models used either a binomial or normal distribution, depending on the data. All models were extended to account for, and test for, an intervention-effect interaction with each severity of intellectual disabilities, and accommodation type. The number of serious adverse events was recorded. No interim analyses or unplanned analyses were undertaken. All analyses were done with SAS version 9.2, and a $5 \%$ significance level was used throughout; all tests were two-sided.

Cost-effectiveness was estimated in terms of incremental cost per quality-adjusted life-year (QALY) gained over 9 months from an NHS perspective, including primary care, drugs, and emergency visits or calls. Unit costs were taken from the Personal Social Services Research Unit (2011), the British National Formulary, and NHS reference costs. The intervention cost was the nurse's time (mean 48 min, range 18-65); we were conservative in costing this for $1 \mathrm{~h}$. No screening costs, or home visit checks were costed because these were assumed to be individual occurrences, with the standard being a health check by a nurse in the GP surgery (the standard rate per week for the practice nurse, as taken from standard reference costs, does allow for a portion of their time allocated to home visits). A within-trial measure of QALYs was identified from EQ-5D scores measured at baseline, 3, 6, and 9 months, using the area-under-the-curve method. ${ }^{26}$ The effect of the intervention is the difference in mean change in QALYs in the alternative groups, taking into account the baseline level of utility. Resource use focused on the health check, and subsequent changes in health-care use including drug use and health-care consultations. We investigated the uncertainty surrounding estimates of cost and effects through bootstrapping by use of a two-stage non-parametric bootstrap ${ }^{27}$ to allow for clustering and correlation of costs and effects, because randomisation was at the GP rather than individual level. (Bootstrapping is a statistical method used to assess the uncertainty in an estimate, which involves resampling and replacement from the original data.) The results are presented graphically on the incremental cost-effectiveness plane; uncertainty about the cost-effectiveness of the intervention is summarised with a cost-effectiveness acceptability curve. The two-stage nonparametric bootstrap ${ }^{27}$ was done with the STATA program version 12. All other analysis and modelling used the MS Excel program. Discounting was not applied because calculations related to a period of less than 1 year. This trial is registered with Current Controlled Trials, number ISRCTN43324841.

\section{Role of the funding source}

The funder of the study had no role in study design, data collection or analysis, data interpretation, or preparation of the manuscript. S-AC had full access to all study data and had final responsibility for the decision to submit for publication.

\section{Results}

Recruited practices were large, medium, and small in size, and had a range of registered patients with intellectual disabilities, so were representative for the area. 38 practices were randomly assigned to either standard care only (control group, $n=67$ patients) or standard care plus practice nurse-delivered health checks (intervention group, $\mathrm{n}=85$ patients); five practices recruited no patients to the trial. 65 $(76 \%)$ of 85 participants in the intervention group received the intervention (figure 1). 66 (99\%) of 67 patients in the control group and $83(98 \%)$ of 85 patients in the intervention group completed the 9 month outcome assessment. Attempts to mask the research assistants during the trial were compromised because some participants revealed their group allocation. 
Table 1 shows participants' baseline characteristics. These did not differ from the wider population with intellectual disabilities living in the same area (median age 44 years [IQR 34-54], sex distribution of $55 \%$ men to $45 \%$ women, $38 \%$ living with a family carer, and $45 \%$ living with a paid carer. Appendix 1 shows health status and number of GP appointments for the preceding 9 months; as expected, the two groups seem balanced.

Only $65(76.5 \%)$ of the health-check group received the intervention (appendix 2 ). One person in the control group received a health check; he was the only enrolled participant at that particular GP practice. Delivery of the health check to this patient was the practice nurse's own initiative; she did not receive health-check materials or training from the investigators.

Table 2 shows health outcomes 9 months after randomisation. All except three participants completed the 9 month data collection. Tables 3 and 4 display the types of detected health needs, and the percentage of these needs that were met. More new health needs were met in the intervention group than in the control group, but this difference was not significant. The health-monitoring needs of longterm disorders were significantly better met in the intervention group than in the control group (table $5)$. Few health-promotion needs in the 9 months after randomisation were addressed in either group (table 6).

General health improved in the intervention group compared with the control group according to EQ5D health-utility scores ( 0.15 vs 0.00 , treatment effect 0.11 [95\% CI $0.02-0.19] \mathrm{p}=0.015)$ between baseline and 9 months after randomisation, but not for SF-36 scores (0.06 vs 0.02 , treatment effect 0.02 [ -0.03 to 0.07] $\mathrm{p}=0.354)$. No serious adverse events attributable to the intervention were recorded.

The within-trial economic analysis showed that the intervention was both more effective $(0.0298$ versus -0.0029), with respect to the mean change in QALYs in the alternate groups (incremental difference 0.0327 [95\% CIs -0.05 to 0.11 ]), and cheaper (incremental difference of $£ 51$ [ -434 to 362]) than standard care from an NHS perspective (appendix). The cost of the intervention was the nurse's time to undertake the health check, which we costed at $£ 51$ per h. ${ }^{28}$ Figure 2 shows the uncertainty surrounding the incremental cost and incremental effect associated with the intervention. There is much uncertainty surrounding the existence and extent of the cost difference, and whether health checks are more effective and the extent of the effect difference, although on average health checks were cheaper and more effective. The cost-effectiveness acceptability curve (figure 3 ) shows the probability that the health-check intervention is cost effective for any cost-effectiveness threshold. The probability that health checks are cost effective is between 0.6 and 0.8 , irrespective of the costeffectiveness threshold level. We also undertook a threshold analysis to explore the maximum value that the intervention could cost so that nurse checks remained dominant compared with standard care. The results of this analysis showed that the intervention cost could rise to from $£ 51$ to $£ 95$ per person before the intervention no longer dominated standard care. Moreover, we were conservative in costing $1 \mathrm{~h}$ of a nurse's time to deliver the health check when the average time was $48 \mathrm{~min}$.

We did not note any significant interaction of ability level, nor accommodation type, for the group difference in proportions of health needs met, health-monitoring needs met, or health-promotion needs met. The one exception was that the benefit of the intervention compared with the control group-with respect to health-monitoring needs being met-was greater for people who lived independent of carers $(p=0.038)$. However, the study was not powered to detect intervention-effect heterogeneity between subgroups.

\section{Discussion}

Practice nurse-delivered health-check interventions for adults with intellectual disabilities were associated with significantly more health monitoring needs being met and generally higher health ratings than standard care for this population. Additionally, more newly detected health needs were met in the intervention group than in the control group, but the difference was not significant. The number of health-promotion needs being met did not differ between groups, which was low in both groups. The economic evaluation showed health checks to be both more effective and cost-saving during the trial period than standard care. As such, the use of health checks in this population dominated usual care. 
Findings are both similar to and different from the Australian GP-delivered health-check trials. ${ }^{20,22}$ These noted a non-significant trend towards new disease detection after 1 year, but they did not report the extent to which needs had been met or obtain data about health monitoring needs. Unlike our trial, they showed an increase in health-promotion activity, especially with testing of vision and hearing, giving tetanus boosters, and checking hepatitis B serology; in our trial the nurses enquired about vision difficulties and testing, then directed people towards an optometrist, rather than directly testing vision, and the health check did not include hepatitis B serology, so data are not similar.

The intervention was less effective than the previous Scottish intervention study, ${ }_{1}^{18}$ in which health checks were undertaken by intellectual disabilities nurses rather than practice nurses, and took about five times longer. The follow-up duration was also longer in that study ( 1 year) compared with our study ( 9 months). The methods for the Scottish intervention study ${ }^{18}$ had limitations, however, because it was not randomised.

Similar to two previous intervention cost reports, the health-check intervention in our study was inexpensive, with the cost of the intervention mainly offset by savings in drug costs (appendix). The intervention cost was the nurse's time (mean $48 \mathrm{~min}$ ); we were conservative in costing this at $1 \mathrm{~h}$. The $£ 100$ paid to participating practices was not included in costing the intervention as we were estimating the cost-effectiveness of the approach if introduced in the NHS rather than in a trial setting. This payment covered the administrative time to accommodate the research assistants accessing information from participant records for the trial. We are unable to disentangle the effect that this payment could have had on participation; however, we suspect that this would only affect the choice to participate rather than the effectiveness of the intervention once individuals had participated and, as such, it would not necessarily affect the cost-effectiveness but rather scale of uptake if initiated.

Improved health care (ie, better addressing of health-monitoring needs) is an important finding. QOF items are evidence-based, and provide pathways to clinically significant benefits for adults with intellectual disabilities who have health disorders. Significantly more health monitoring needs were addressed in the intervention group than in the control group, including management of long-term chronic conditions such as hypertension and diabetes, and it is well established that such management improves health. This finding is especially important in view of the barriers that adults with intellectual disabilities experience when trying to access primary care. ${ }^{4}$ This finding replicates results reported in the Scottish intervention study. ${ }^{18}$ Detection and addressing of unmet needs is also important because early detection could lead to better outcomes; our study might have been underpowered for this outcome, having used the same power as the previous Scottish intervention study. ${ }^{18} \mathrm{~A}$ better outcome in the intervention group was noted for the EQ-5D. Longer-term follow-up is necessary to investigate further the differences in health outcomes between the two groups.

Practice nurses are very familiar with QOF items. Other highly prevalent, but often overlooked, disorders for adults with intellectual disabilities were only infrequently detected in our study. These disorders might already have been identified and addressed, or might not have been present in our study participants. Another interpretation is that the practice nurses, unfamiliar with the presentation and importance of these disorders in this population, and despite prompts by the health-check instrument, did not identify them. Two previous studies drew similar conclusions. ${ }^{29,30} \mathrm{~A}$ study of healthcheck findings in 27 English practices showed processes incentivised through the QOF were completed in more health-checked individuals than those receiving standard care, but processes more specific to intellectual disabilities were poorly recorded for all. ${ }^{29}$ An assessment of the English health-check programme in 171 practices also reported this result. ${ }^{30}$ Educational approaches might therefore be of benefit, and joint learning by intellectual disabilities nurses and practice nurses could offer an innovative model.

The study was powered from the Scottish intervention study. ${ }^{18}$ During the study, it soon became apparent that the mean number of participants recruited per practice would be greater than the 2.5 originally assumed. However, initial (masked) assessment of outcomes suggested that the level of clustering of outcomes was very small $(<0.1)$, suggesting that clustering of outcomes would not affect sample size. However, with respect to the primary outcome, the proportion reported in the control group (73\%) was much higher than assumed in the sample size calculation (33\%), so with the sample size achieved, we would have needed the primary outcome rate to be $92 \%$ in the intervention group (we noted $76 \%$ ) to have had at least $80 \%$ power in detecting a difference. 
The reasons for people not receiving a health check in the interventions group underline the pressures in primary care that result in inflexibility. Therefore, the $76 \%$ in the intervention group that received the health check is actually quite encouraging. In an NHS service (rather than this study, for which the intervention was one health check undertaken by a practice nurse), individuals who do not attend appointments could be followed up by a community intellectual disabilities team visit to find out why not, establish if any reasonable adjustments are needed, and help to facilitate the appointment. Community teams have a recognised role in supporting access to primary care, and such actions could be refocused. Because paid carers in the UK have a duty to address health care, if paid carers state that they are too busy to attend such health checks (as two in this study did), this could be drawn to the attention of service-provider managers for resolution, because it could be construed as breach of contract. The standard rate per $\mathrm{h}$ for the practice nurse, as taken from standard reference costs, does allow for a portion of their time allocated to home visits, though none were undertaken in this trial.

The participants' age range did not differ from that of the adult intellectual disabilities population; as expected, men outnumbered women (because more boys are born with intellectual disabilities than girls) and a range of accommodation types were reported. Participant characteristics were similar to the population with intellectual disabilities living in the same geographical area. The Greater Glasgow and Clyde area of Scotland in which this trial took place includes a mix of urban and semirural areas, and large and small practices that have between 0 and 80 registered patients with intellectual disabilities. We restricted the number recruited from each practice to include more practices and to capture a range of attitudes and skills in primary care, and the balance achieved does not differ from characteristics of practices in the area. These study findings are therefore generalisable within the UK NHS, but perhaps not in countries where primary health-care systems are differently organised and delivered.

Our study has several limitations. In retrospect, it was probably underpowered to detect a difference for the primary outcome measure because of the higher-than-expected proportion of health needs noted in the control group, compared with the previous Scottish intervention study which was similarly powered. The EQ-5D and SF-36 might not measure the health dimensions that adults with intellectual disabilities regard as most important. There could be inconsistency in whether people rate intellectual disabilities as a health problem. Some ratings were necessarily by proxy, and might not show the views of the adult with intellectual disabilities, and, because of staff turnover, baseline and follow-up measures were not always completed with the same carer. Research into the use of these instruments with this population is scarce, ${ }^{31}$ and we are not aware of other studies using them to measure changes in health status over time. Additionally, the economic evaluation excluded hospital costs with the exception of Accident and Emergency department costs. The intervention was delivered in primary care and these services were the most frequently accessed by both groups in a 12 month period in a previous economic investigation into the costs of a health check for people with intellectual disabilities. ${ }^{19}$ The results of that investigation also suggested that expanding the cost perspective beyond the NHS might strengthen cost-effectiveness because of savings in non-medical and social services, although these findings were not significant. No adverse events related to the intervention were reported in our trial but only 152 participants were included, so adverse events might occur in a larger population.

Research in this discipline is scarce; however, investments have still been made in GP-delivered health checks for adults with intellectual disabilities in some countries (such as England and Wales), although not in others (eg, Scotland). This practice nurse-delivered health check was effective in some important domains, and cost effective. Its outcomes were either similar or favourable compared with the Australian GP-delivered health check. ${ }^{20}$ Are health checks performed better by practice nurses or intellectual disabilities nurses? Intellectual disabilities nurses have skills in the health-need profile, communication skills with adults with intellectual disabilities, and experience of working with carers. Practice nurses have skills in general health, and experience of working with GPs, and monitoring and managing disease. These skill sets are complementary, and perhaps suggest the benefits of joint working. Health checks delivered by practice nurses are indicated for this population, who typically would otherwise have poor access to primary care.

\section{Contributors}

S-AC designed the study, oversaw all aspects of study implementation, and prepared the report with assistance from NG. JM, LMA, AM, CAM, GM, EADG, and EF helped with study design and implementation and approved the final version of the report. JM, LMA, AM, MCB, LAM, CL, GM, EADG, 
and EF also contributed intellectual content to the report. AM led the statistical analyses with assistance from S-AC and NG. MCB, LAM, and CL acquired the data. The health economics design and analyses were led by EADG and supervised by EF.

\section{Declaration of interests}

We declare no competing interests.

\section{Acknowledgments}

This study was funded by the Scottish Government Change Fund and NHS Greater Glasgow and Clyde Research and Development.

\section{Research in context \\ Systematic review}

People with intellectual disabilities experience substantial health inequalities compared with the general population. Clinical trials, current controlled trials, and an international clinical trials registry platform were searched. Additionally, we identified a systematic review that searched Medline, CINAHL, Web of Science, and PsycINFO. ${ }^{17}$ Results from the review showed very little previous evidence, with no previous controlled trials of practice nurse-delivered health checks for people with intellectual disabilities, and only two controlled trials identified of GP-delivered health checks in Australia (that showed some increase in health-promotion activities).

\section{Interpretation}

This is the first randomised controlled trial of practice nurse-delivered health checks for adults with intellectual disabilities. The intervention produced better general health ratings and health-care improvements, which might improve longer-term health compared with standard care, but not the number of new health needs met during 9 months; it was also cheaper and more effective. Health checks designed for people with intellectual disabilities are needed, rather than health checks designed for the general population without selection for risk factors or disease.

\section{References}

1 Tyrer F, Smith LK, McGrother CW. Mortality in adults with moderate to profound intellectual disability: a population-based study. J Intellect Disabil Res 2007; 51: 520-27.

2 Cooper S-A, Smiley E, Morrison J, Williamson A, Allan L. Mental ill-health in adults with intellectual disabilities: prevalence and associated factors. Br J Psychiatry 2007; 190: 27-35.

3 Jones S, Cooper S-A, Smiley E, Allan L, Williamson A, Morrison J. Prevalence of, and factors associated with, problem behaviours in adults with intellectual disabilities. J Nerv Ment Dis 2008; 196: 678-86.

4 NHS Health Scotland. People with learning disabilities in Scotland: the health needs assessment report. Glasgow: NHS Scotland, 2004.

5 van Splunder J, Stilma JS, Bernsen RM, Arentz TG, Evenhuis HM. Refractive errors and visual impairment in 900 adults with intellectual disabilities in the Netherlands. Acta Ophthalmol Scand 2003; 81: $123-29$.

6 Warburg M. Visual impairment in adult people with moderate, severe, and profound intellectual disability. Acta Ophthalmol Scand 2001; 79: 450-54.

7 Evenhuis HM. Hearing loss in middle-age persons with Down syndrome. Am J Ment Retard 1992; 97: 47-56.

8 Crandell CC. Roeser RJ. Incidence of excessive/impacted cerumen in individuals with mental retardation: a longitudinal investigation. Am J Ment Retard 1993; 97: 568-74. 
9 Böhmer CJ, Niezen-de Boer MC, Klinkenberg-Knol EC, Deville WL, Nadorp JH, Meuwissen SG. The prevalence of gastroesophageal reflux disease in institutionalized intellectually disabled individuals. $\mathrm{Am}$ J Gastroenterol 1999; 94: 804-10.

10 Finlayson J, Jackson A, Mantry D, Morrison J, Cooper S-A. Injuries, falls, and accidents in the population of adults with intellectual disabilities. J Intellect Disab Res 2010; 54: 966-80.

11 Cooper S-A, Melville C, Morrison J. People with intellectual disabilities. Their health needs differ and need to be recognised and met. BMJ 2004; 329: 41-45.

12 Scheepers M, Kerr M, O'Hara D, et al. Reducing health disparity in people with intellectual disabilities: a report from the Health Issues Special Interest Research Group of the International Association for the Scientific Study of Intellectual Disabilities. J Policy Practice Intellect Dis 2005; 2: 249-55.

13 Lennox $N$, Beange $H$, Parmenter $T$, et al. Health guidelines for adults with an intellectual disability. International Association for the Scientific Study of Intellectual Disabilities 2002. http://iassid. org/pdf/healthguidelines-2002.pdf (accessed Nov 10, 2014).

14 Mencap. Death by indifference 2007 http://www.mencap.org.uk/ document.asp?id=284 (accessed Nov 10, 2014).

15 Disability Rights Commission. Equal treatment: closing the gap. A formal investigation into the health inequalities experienced by people with learning disabilities and/or mental health problems.

2006. http://disability-studies.leeds.ac.uk/files/library/DRCHealth- FI-main.pdf (accessed Nov 10, 2014).

16 Krogsbøll, LT, Jørgensen KT, Larson CG, Grønhøj PC. General health checks in adults for reducing morbidity and mortality from disease. Cochrane systematic review and meta-analysis. BMJ 2012;

345: e7191.

17 Robertson J, Robert H, Emerson E. Health checks for people with learning disabilities: a systematic review of evidence. Improving Health and Lives: Learning Disabilities Observatory. Durham University, 2010.http://www.improvinghealthandlives.org.uk/uploads/doc/vid_7646_IHAL201004HealthChecksSys tematicReview.pdf (accessed Nov 10, 2014).

18 Cooper S-A, Morrison J, Melville C, et al. Improving the health of people with intellectual disabilities: outcomes of a health screening programme after one year. J Intellect Disabil Res 2006; 50: 667-77.

19 Romeo R, Knapp M, Morrison J, et al. Cost estimation of a health check intervention for people with intellectual disabilities in the UK. J Intellect Disabil Res 2009; 53: 426-39.

20 Lennox N, Bain C, Rey-Conde T, Purdie D, Bush R, Pandeya N. Effects of a comprehensive health assessment programme for Australian adults with intellectual disability: a cluster randomized trial. Int J Epidemiol 2007; 36: 139-46.

21 Gordon LG, Holden L, Ware RS, Taylor MT, Lennox NG. Comprehensive health assessments for adults with intellectual disability living in the community. Weighing up the costs and benefits. Aust Fam Physician 2012; 41: 969-72.

22 Lennox N, Bain C, Rey-Conde T, et al. Cluster randomized controlled trial of a tailored intervention to improve health for adults with intellectual disability who live in private dwellings. J Appl Res Intellect Dis 2010; 23: 303-11.

23 Byrne JH, Ware RS, Lennox NG. Health actions prompted by health assessments for people with intellectual disability exceed actions recorded in general practitioners records. Aus J Primary Health 2014; published online June 10, 2014. http://dx.doi.org/10.1071/PY14007.

24 Beecham J, Knapp MRJ. Costing psychiatric interventions. In: Thornicroft GJ, Brewin CR, Wing JK, eds. Measuring mental health needs. London: Gaskell, 1992. 
25 Sparrow SS, Balla DA, Cicchetti DV. A revision of the Vineland Social Maturity Scale by EA Doll. Minnesota: American Guidance Service, 1984.

26 Manca A, Hawkins N, Sculpher MJ. Estimating mean QALYs in trial-based cost-effectiveness analysis: the importance of controlling for baseline utility. Health Econ 2005; 14: 487-96.

27 Ng ESW, Grieve R, Carpenter JR. Two-stage nonparametric bootstrap sampling with shrinkage correction for clustered data. Stata J 2013; 13: 141-64.

28 Personal Social Services Research Unit. Unit cost of health and social care. 2011. http://www.pssru.ac.uk/project-pages/unitcosts/2011/index.php (accessed Nov 10, 2014).

29 Chauhan U, Kontopantelis E, Campbell S, Jarrett H, Lester H. Hypothesis: health checks in primary care for adults with intellectual disabilities: how extensive should they be? J Intellect Disabil Res 2010; 54: 479-86.

30 Chauhan U, Reeve J, Evangelos K, Hinder S, Nelson P, Doran T. Impact of the English Directly Enhanced Service (DES) for learning disability. Manchester University, 2012.

31 Riemsma RP, Forbes CA, Glanville JM, Eastwood AJ, Kleijnen J. General health status measures for people with cognitive impairment: learning disability and acquired brain injury. Health Technol Assess 2001; 5: 6. 
Figure 1: Flow diagram

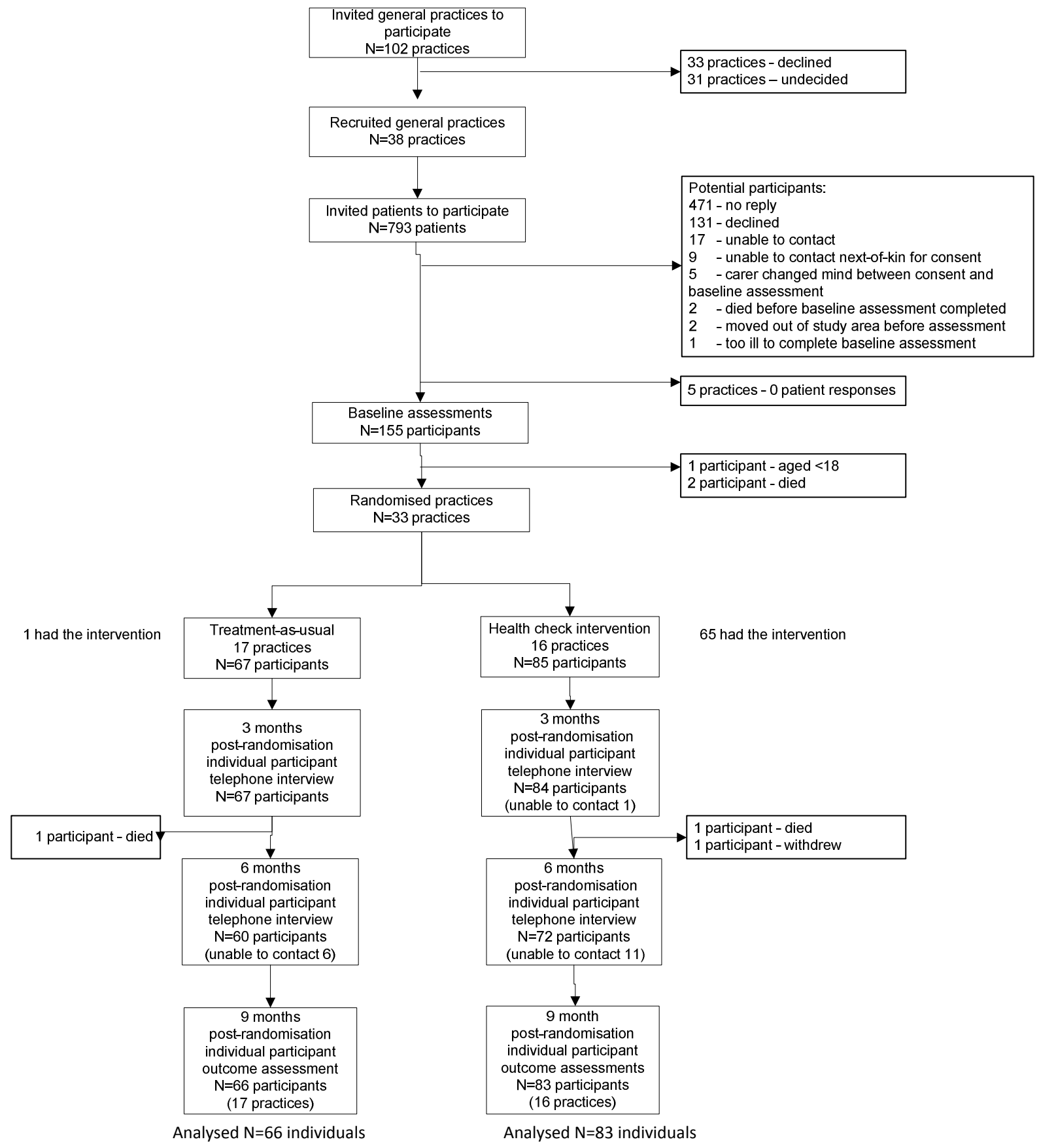


Figure 2: Incremental cost-effectiveness plane for health check compared to usual care

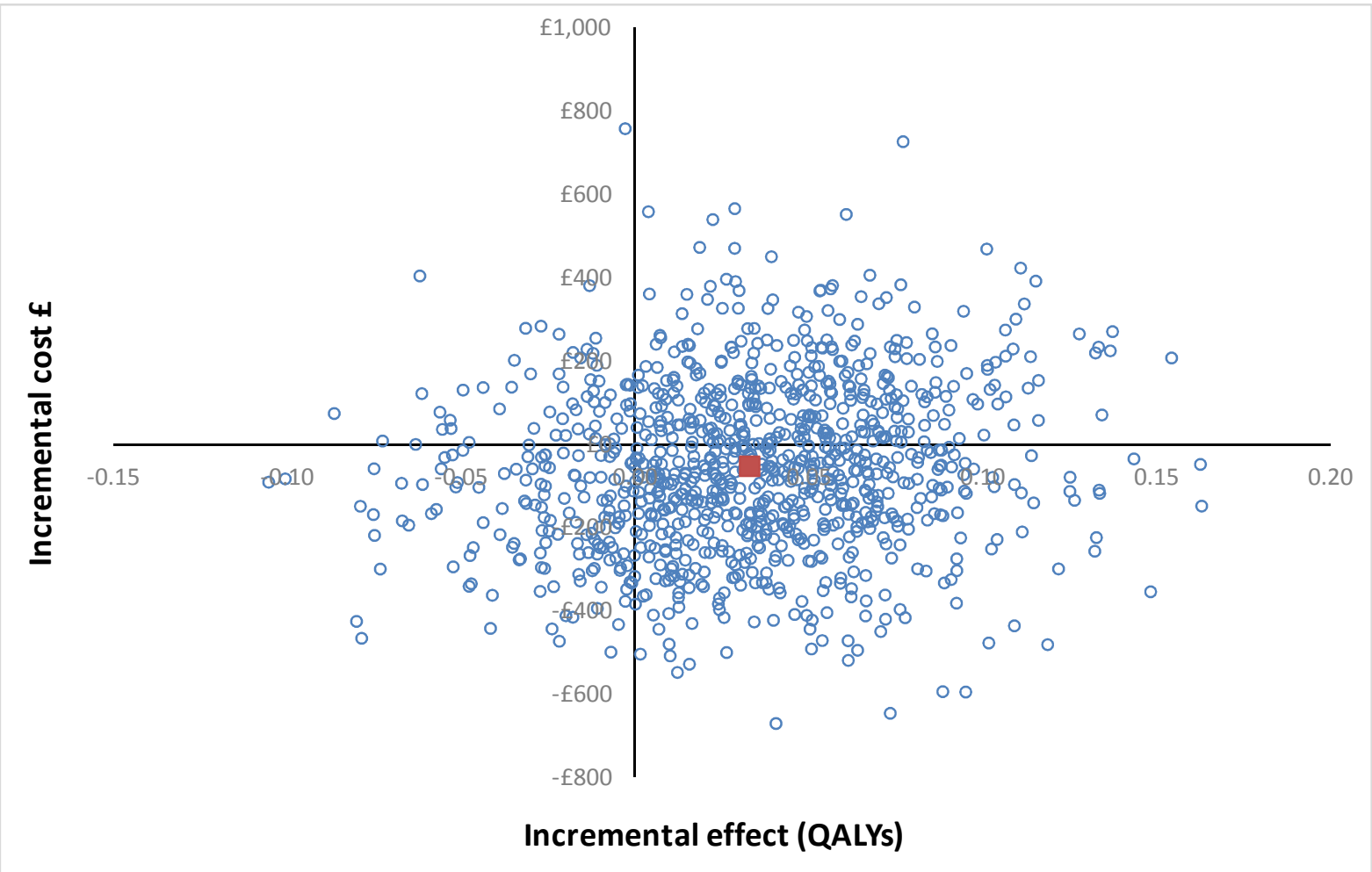

The ICER i.e. the mean cost-effectiveness estimate is denoted by the red dot. 
Figure 3: Cost-effectiveness acceptability curve for health check compared to usual care

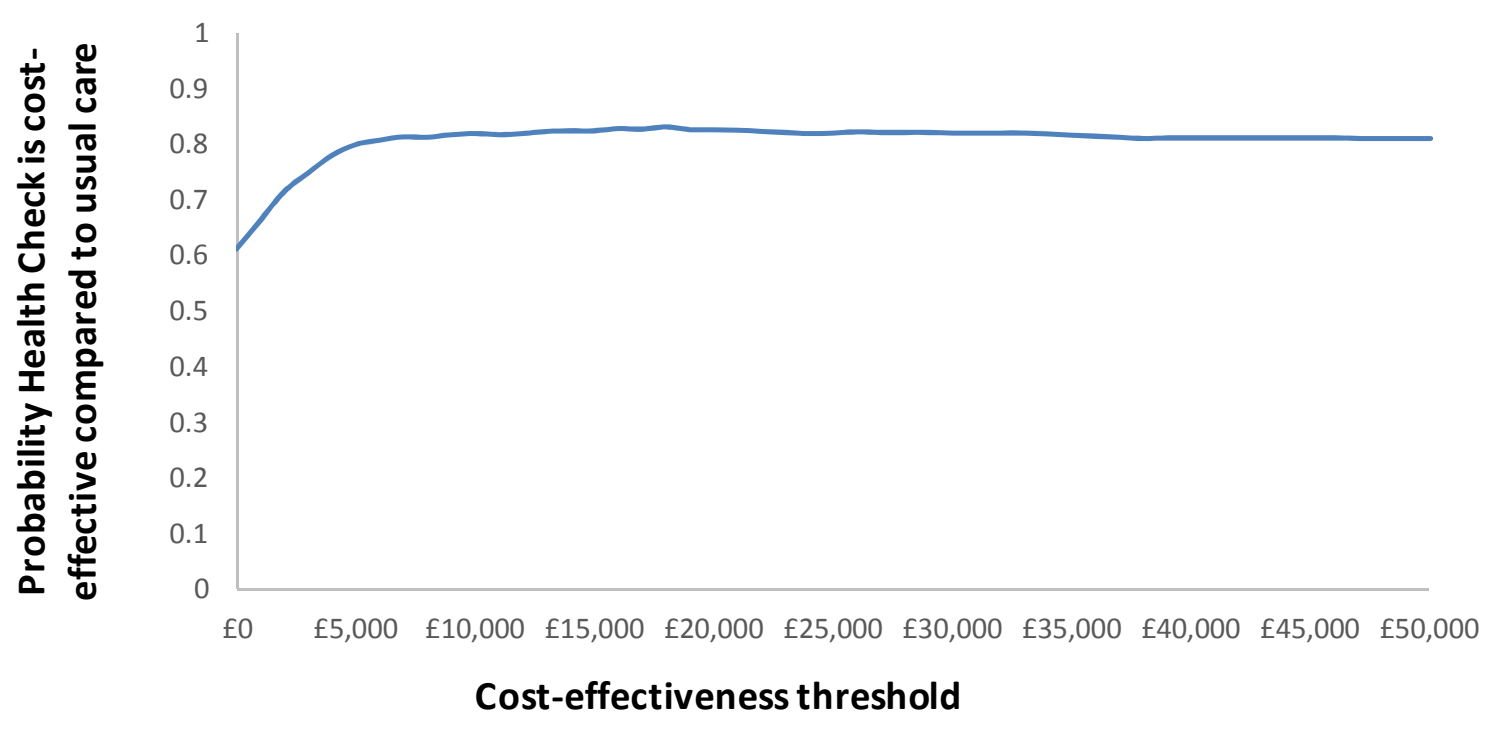

X-axis: Cost-effectiveness willingness to pay to QALYs 
Table 1: Baseline characteristics of participants

$\begin{array}{ll}\begin{array}{l}\text { Control group } \\ (n=67)\end{array} & \begin{array}{l}\text { Health-check } \\ \text { group }(n=85)\end{array}\end{array}$

$\begin{array}{lll}\text { Age (years) } & 46 \cdot 2(38 \cdot 2-57 \cdot 3) & 47 \cdot 2(34 \cdot 5-54 \cdot 6) \\ \text { Women } & 28(42 \%) & 32(38 \%) \\ \text { Disability } & 12(18 \%) & 18(21 \%) \\ \quad \text { Mild intellectual disabilities } & 15(22 \%) & 28(33 \%) \\ \quad \text { Moderate intellectual disabilities } & 23(34 \%) & 26(31 \%) \\ \quad \text { Severe intellectual disabilities } & 17(25 \%) & 13(15 \%) \\ \quad \text { Profound intellectual disabilities } & 27(41 \%) & 34(40 \%) \\ \text { Accommodation } & 30(45 \%) & 36(42 \%) \\ \quad \text { With a family carer } & 9(14 \%) & 15(18 \%) \\ \text { With a paid carer } & \end{array}$

Data are number (\%) or median (IQR range) for the intention-to-treat population. 
Table 2: Health outcomes during the 9 months after randomisation

\begin{tabular}{|c|c|c|c|c|}
\hline & \multirow[t]{2}{*}{$\begin{array}{l}\text { Control group } \\
(n=66)\end{array}$} & \multirow[t]{2}{*}{$\begin{array}{l}\text { Health-check } \\
\text { group }(n=83)\end{array}$} & \multicolumn{2}{|c|}{$\begin{array}{l}\text { Comparison for control vs health } \\
\text { check }\end{array}$} \\
\hline & & & OR $(95 \% \mathrm{Cl})$ & $p$ value \\
\hline \multicolumn{5}{|l|}{ Health needs } \\
\hline Newly detected & $2(0-11)$ & $1(0-8)$ & $1.73(0.93-3.22)$ & 0.085 \\
\hline Mean \% met & $72 \cdot 6 \%(S D 35 \cdot 4)$ & $76 \cdot 4 \%(S D 36 \cdot 5)$ & & \\
\hline \multicolumn{5}{|c|}{ Health-monitoring needs } \\
\hline Newly detected & $2(0-22)$ & $2(0-20)$ & $2 \cdot 38(1 \cdot 31-4 \cdot 32)$ & 0.0053 \\
\hline Mean \% met & $56 \cdot 8 \%(S D 29 \cdot 4)$ & $69 \cdot 9 \%(\mathrm{SD} 34 \cdot 2)$ & & \\
\hline \multicolumn{5}{|c|}{ Health-promotion needs } \\
\hline Newly detected & $6(1-9)$ & $6(1-9)$ & $0.98(0.73-1.32)$ & 0.878 \\
\hline Mean $\%$ met & $35 \cdot 9 \%(S D 23 \cdot 1)$ & $35 \cdot 3 \%(S D 22 \cdot 3)$ & & \\
\hline \multicolumn{5}{|c|}{ Appointments and drugs } \\
\hline GP appointments & $3(0-17)$ & $2(0-16)$ & $-0.66(-1.66 \text { to } 0.34)^{*}$ & 0.191 \\
\hline Medicines needed & $7(0-26)$ & $5(0-22)$ & $0.41(-0.70 \text { to } 1.51)^{*}$ & 0.467 \\
\hline \multicolumn{5}{|c|}{$\begin{array}{l}\text { Data are median (min-max). OR=odds ratio. GP=general practitioner. }{ }^{*} \text { Effect estimate }(95 \% \mathrm{Cl} \text { ) reported is adjusted mean } \\
\text { difference for the change in the number of GP appointments, or the change in the number of drugs needed, at } 9 \text { months } \\
\text { from baseline. }\end{array}$} \\
\hline
\end{tabular}


Table 3: Types of health needs detected and treated during 9 months after randomisation

\begin{tabular}{|c|c|c|c|c|}
\hline & \multicolumn{2}{|c|}{ Control group $(n=66)$} & \multicolumn{2}{|c|}{ Health-check group $(n=83)$} \\
\hline & $\begin{array}{l}\text { Number of } \\
\text { events } \\
\text { detected }\end{array}$ & $\begin{array}{l}\text { Number of events } \\
\text { treated/number of } \\
\text { events detected (\% } \\
\text { of detected needs } \\
\text { treated) }\end{array}$ & $\begin{array}{l}\text { Number of } \\
\text { events } \\
\text { detected }\end{array}$ & $\begin{array}{l}\text { Number of events } \\
\text { treated/number of } \\
\text { events detected (\% } \\
\text { of detected needs } \\
\text { treated) }\end{array}$ \\
\hline Infections & 7 & $7 / 7(100 \%)$ & 3 & $3 / 3(100 \%)$ \\
\hline Neoplasm & 0 & 0 & 0 & 0 \\
\hline Blood disorders & 0 & 0 & 2 & $2 / 2(100 \%)$ \\
\hline Endocrine disorders & 4 & $4 / 4(100 \%)$ & 1 & $1 / 1(100 \%)$ \\
\hline Mental disorders & 14 & $7 / 14(50 \%)$ & 10 & $4 / 10(40 \%)$ \\
\hline Nervous system & 24 & $7 / 24(29 \%)$ & 17 & $4 / 17(24 \%)$ \\
\hline Eye disorders & 5 & $4 / 5(80 \%)$ & 2 & $2 / 2(100 \%)$ \\
\hline Ear disorders & 6 & $6 / 6(100 \%)$ & 15 & $15 / 15(100 \%)$ \\
\hline Circulatory disorders & 9 & $5 / 9(56 \%)$ & 14 & $7 / 14(50 \%)$ \\
\hline Respiratory disorders & 14 & $14 / 14(100 \%)$ & 15 & $15 / 15(100 \%)$ \\
\hline Gastrointestinal disorders & 6 & $5 / 6(83 \%)$ & 11 & $11 / 11(100 \%)$ \\
\hline Skin disorders & 20 & $20 / 20(100 \%)$ & 24 & $23 / 24(96 \%)$ \\
\hline Musculoskeletal disorders & 5 & $4 / 5(80 \%)$ & 15 & $14 / 15(93 \%)$ \\
\hline Genito-urinary disorders & 17 & $15 / 17(88 \%)$ & 5 & $5 / 5(100 \%)$ \\
\hline Pregnancy & 0 & 0 & 0 & 0 \\
\hline Perinatal disorders & 0 & 0 & 0 & 0 \\
\hline Congenital disorders & 0 & 0 & 0 & 0 \\
\hline Other disorders & 6 & $3 / 6(50 \%)$ & 1 & $0 / 1(0 \%)$ \\
\hline Injury or poisoning & 5 & $5 / 5(100 \%)$ & 2 & $1 / 2(50 \%)$ \\
\hline External causes & 0 & 0 & 0 & 0 \\
\hline
\end{tabular}


Table 4: Most often reported health needs detected and treated during 9 months after randomisation

\begin{tabular}{|c|c|c|c|c|}
\hline & \multicolumn{2}{|c|}{ Control group $(n=66)$} & \multicolumn{2}{|c|}{ Health-check group $(n=83)$} \\
\hline & $\begin{array}{l}\text { Number of events } \\
\text { detected }\end{array}$ & $\begin{array}{l}\text { Number of events } \\
\text { treated/number of } \\
\text { events detected (\% } \\
\text { of detected needs } \\
\text { treated) }\end{array}$ & $\begin{array}{l}\text { Number of events } \\
\text { detected }\end{array}$ & $\begin{array}{l}\text { Number of events treated } / \text { number } \\
\text { of events detected (\% of detected } \\
\text { needs treated) }\end{array}$ \\
\hline $\begin{array}{l}\text { Outstanding blood tests (excluding tests } \\
\text { included in the QOF) }\end{array}$ & 23 & $8 / 23(35 \%)$ & 16 & $6 / 16(38 \%)$ \\
\hline Skin rash, psoriasis, or eczema & 12 & $12 / 12(100 \%)$ & 20 & $18 / 20(90 \%)$ \\
\hline Chest infection & 13 & $13 / 13(100 \%)$ & 12 & $12 / 12(100 \%)$ \\
\hline Urinary tract infection & 13 & $12 / 13(92 \%)$ & 3 & $3 / 3(100 \%)$ \\
\hline Injury & 6 & $5 / 6(83 \%)$ & 8 & $6 / 8(75 \%)$ \\
\hline Cellulitis or ulcers & 9 & $9 / 9(100 \%)$ & 3 & $3 / 3(100 \%)$ \\
\hline Fungal infection & 3 & $3 / 3(100 \%)$ & 7 & $7 / 7(100 \%)$ \\
\hline All other reported health needs & 63 & $44 / 63(70 \%)$ & 68 & $52 / 68(77 \%)$ \\
\hline
\end{tabular}


Table 5: Long-term disorders during 9 months after randomisation and relevant QOF items for each disorder

\begin{tabular}{|c|c|c|c|c|}
\hline & \multicolumn{2}{|l|}{ Control group $(n=66)$} & \multicolumn{2}{|c|}{ Health-check group $(n=83)$} \\
\hline & $\begin{array}{l}\text { Number of participants } \\
\text { with each health need } \\
(\%)\end{array}$ & $\begin{array}{l}\text { Median number of QOF items } \\
\text { that were treated/ median } \\
\text { number of items that were } \\
\text { applicable (median \% of items } \\
\text { that were treated) }\end{array}$ & $\begin{array}{l}\text { Number of participants } \\
\text { with each health need } \\
(\%)\end{array}$ & $\begin{array}{l}\text { Median number of QOF items that } \\
\text { were treated/median number of } \\
\text { items that were applicable (median } \\
\% \text { of items that were treated) }\end{array}$ \\
\hline Coronary heart disease & $1(1.5 \%)$ & $3 / 9(33 \cdot 3 \%)$ & $4(4 \cdot 8 \%)$ & $5 / 6(90 \%)$ \\
\hline Heart failure & $0(0 \%)$ & $0(0 \%)$ & $0(0 \%)$ & $0(0 \%)$ \\
\hline Stroke or TIA & $1(1.5 \%)$ & $4 / 5(80 \%)$ & $1(1 \cdot 2 \%)$ & $7 / 7(100 \%)$ \\
\hline Hypertension & $7(10 \cdot 6 \%)$ & $1 / 2(50 \%)$ & $16(19 \cdot 3 \%)$ & $2 / 2(100 \%)$ \\
\hline Diabetes & $7(10 \cdot 6 \%)$ & $9 / 14(64 \%)$ & $8(9.6 \%)$ & $10 \cdot 5 / 12(85 \%)$ \\
\hline COPD & $1(1.5 \%)$ & $2 / 4(50 \%)$ & $0(0 \%)$ & $0(0 \%)$ \\
\hline Epilepsy & $21(31 \cdot 8 \%)$ & $2 / 3(67 \%)$ & $17(20 \cdot 5 \%)$ & $1 / 3(33 \%)$ \\
\hline Hypothyroidism & $6(9 \cdot 1 \%)$ & $1 / 1(100 \%)$ & $10(12 \cdot 0 \%)$ & $1 / 1(100 \%)$ \\
\hline Psychosis & $6(9 \cdot 1 \%)$ & $0.5 / 2(25 \%)$ & $4(4 \cdot 8 \%)$ & $0 / 1.5(0 \%)$ \\
\hline Asthma & $4(6 \cdot 1 \%)$ & $0.5 / 1(50 \%)$ & $11(13 \cdot 3 \%)$ & $0 / 1(0 \%)$ \\
\hline Atrial fibrillation & $0(0 \%)$ & $0(0 \%)$ & $0(0 \%)$ & $0(0 \%)$ \\
\hline Chronic kidney disease & $1(1.5 \%)$ & $2 / 3(67 \%)$ & $2(2 \cdot 4 \%)$ & $3 / 3(100 \%)$ \\
\hline Smoking domain & $9(14 \cdot 1 \%)$ & $1 / 1(100 \cdot 0 \%)$ & $27(32.5 \%)$ & $1 / 1(100 \%)$ \\
\hline Depression screening & $6(9 \cdot 4 \%)$ & $0 / 1(0 \%)$ & $11(13 \cdot 3 \%)$ & $1 / 1(100 \%)$ \\
\hline
\end{tabular}

Data are $n(\%)$ of participants with each health need; median number of QOF items that were treated/median number of QOF items that were applicable (median \% of items that were treated). QOF=GP Quality and Outcomes Framework. TIA=transient ischaemic attack. COPD=chronic obstructive pulmonary disease. 
Table 6: Health-promotion needs during the 9 months after postrandomisation

\begin{tabular}{|c|c|c|c|c|}
\hline & \multicolumn{2}{|c|}{ Control group $(n=66)$} & \multicolumn{2}{|c|}{ Health-check group $(n=83)$} \\
\hline & $\begin{array}{l}\text { Number of events } \\
\text { detected }\end{array}$ & $\begin{array}{l}\text { Number of events treated/ } \\
\text { number of events detected } \\
\text { (\% of detected needs treated) }\end{array}$ & $\begin{array}{l}\text { Number of events } \\
\text { detected }\end{array}$ & $\begin{array}{l}\text { Number of events treated/number } \\
\text { of events detected (\% of detected } \\
\text { needs treated) }\end{array}$ \\
\hline Smoking & 4 & $2 / 4(50 \%)$ & 9 & $5 / 9(56 \%)$ \\
\hline Alcohol excess & 0 & 0 & 0 & 0 \\
\hline Illicit drug use & 0 & 0 & 1 & $1 / 1(100 \%)$ \\
\hline Obesity & 39 & $10 / 39(26 \%)$ & 57 & $21 / 57(37 \%)$ \\
\hline Cervical screening & 20 & $6 / 20(20 \%)$ & 27 & $5 / 27(19 \%)$ \\
\hline Mammography & 11 & $4 / 11(36 \%)$ & 15 & $6 / 15(40 \%)$ \\
\hline Tetanus immunisation & 62 & $13 / 62(21 \%)$ & 80 & $11 / 80(14 \%)$ \\
\hline Polio immunisation & 62 & $11 / 62(18 \%)$ & 79 & $12 / 79(15 \%)$ \\
\hline Influenza immunisation & 44 & $27 / 44(61 \%)$ & 48 & $34 / 48(71 \%)$ \\
\hline Hepatitis $B$ vaccination & 24 & $2 / 24(8 \%)$ & 27 & $3 / 27(11 \%)$ \\
\hline Dental check-up & 63 & $43 / 63(68 \%)$ & 80 & $41 / 80(51 \%)$ \\
\hline Vision test & 0 & 0 & 0 & 0 \\
\hline Hearing test & 0 & 0 & 0 & 0 \\
\hline Sex and relationship information & 0 & 0 & 0 & 0 \\
\hline $\begin{array}{l}\text { Thyroid function tests (Down } \\
\text { syndrome) }\end{array}$ & 7 & $6 / 7(86 \%)$ & 14 & $8 / 14(57 \%)$ \\
\hline Being sedentary & 63 & $20 / 63(32 \%)$ & 80 & $27 / 80(34 \%)$ \\
\hline Being underweight & 0 & 0 & 2 & $1 / 2(50 \%)$ \\
\hline Other & 1 & $0 / 1(0 \%)$ & 0 & 0 \\
\hline
\end{tabular}

Data are number of health-promotion needs detected (\%) and number of detected health promotion needs treated/ health-promotion needs detected. 
Appendix 1: Health service usage and health status for the nine months prior to baseline

\begin{tabular}{|c|c|c|}
\hline & $\begin{array}{l}\text { Control group } \\
N=67\end{array}$ & $\begin{array}{l}\text { Health check group } \\
\mathrm{N}=85\end{array}$ \\
\hline $\begin{array}{l}\text { Health needs } \\
\text { Newly detected } \\
\text { Mean percentage met }\end{array}$ & $\begin{array}{c}1(0-17) \\
74 \cdot 7\end{array}$ & $\begin{array}{l}1(0-17) \\
73 \cdot 3\end{array}$ \\
\hline $\begin{array}{l}\text { Health monitoring needs } \\
\text { Newly detected } \\
\text { Mean percentage met }\end{array}$ & $\begin{array}{l}2(0-23) \\
57.9\end{array}$ & $\begin{array}{l}2(0-24) \\
61 \cdot 8\end{array}$ \\
\hline $\begin{array}{l}\text { Health promotion needs } \\
\text { Newly detected } \\
\text { Mean percentage met }\end{array}$ & $\begin{array}{c}6(3-8) \\
41 \cdot 1\end{array}$ & $\begin{array}{c}6(4-9) \\
36 \cdot 4\end{array}$ \\
\hline GP appointments & $3(0-16)$ & $2(0-27)$ \\
\hline Medication & $6(0-24)$ & $4(0-21)$ \\
\hline
\end{tabular}

Summaries reported are either the median (minimum, maximum) or the mean percentage met. 
Appendix 2: Delivery of intervention

\begin{tabular}{|l|l|l|}
\hline & $\begin{array}{l}\text { Standard care group } \\
\mathbf{N = 6 7}\end{array}$ & $\begin{array}{l}\text { Health check group } \\
\mathbf{N = 8 5}\end{array}$ \\
\hline Health checks completed & $1(1 \cdot 5)$ & $65(76 \cdot 5)$ \\
\hline Reason for no health check & $66(98 \cdot 5)$ & \\
Control group & & $8(9 \cdot 4)$ \\
Sole practice nurse went on sick leave & & $6(7 \cdot 1)$ \\
Sent appointment but didn't attend & & $2(2 \cdot 4)$ \\
Paid carers said they were too busy & & $2(2 \cdot 4)$ \\
No appointment sent & $1(1 \cdot 2)$ \\
Too depressed to attend the practice & & $1(1 \cdot 2)$ \\
Appointment offered clashed with & & \\
college course & & \\
\hline
\end{tabular}

Summaries reported are the number of participants (percentage) 
